\title{
NOVEL PROCEDURE TO COMPUTE A CONTACT ZONE MAGNITUDE OF VIBRATIONS OF TWO-LAYERED UNCOUPLED PLATES
}

\author{
J. AWREJCEWICZ, V. A. KRYSKO, AND O. OVSIANNIKOVA
}

Received 15 February 2004

A novel iteration procedure for dynamical problems, where in each time step, a contacting plates' zone is improved, is proposed. Therefore, a zone and magnitude of a contact load are also improved. Investigations of boundary conditions' influence on externally driven vibrations of uncoupled two-layer plates, where for each of the layers, the Kirchhoff hypothesis holds, are carried out.

\section{Introduction}

Uncoupled multilayer plates create a complex dynamical structure, where depending on input parameters and initial and boundary conditions, various vibration types appear.

In spite of undoubtable achievements in static and dynamical problems of nonlinear theory of plates, the problems of contacts exhibited by multilayer plates subject to both longitudinal and transversal time-changeable loads are less investigated.

\section{Mathematical models}

In this work, a model of two-layer construction composed of thin elastic rectangular plates is studied [7]. The mean surface of an upper plate lies in plane $z=0$, whereas the mean surface of the lower one lies in plane $z=(1 / 2)\left(\delta_{1}+\delta_{2}\right)+h_{1}$, where $h_{1}$ is the distance between plates, $\delta_{1}, \delta_{2}$ are the thicknesses of upper and lower plates, correspondingly. The plates are contacting with each other through external surfaces, projected into a corresponding mean surface, within a general Winkler's hypothesis [5]. Note that each of the plates is embedded in $3 \mathrm{D}$ space in $\mathbb{R}^{3}$ with attached coordinates. Namely, senses of the axes $O x$ and $O y$ go into direction of mean surface of upper plate, whereas axis $\mathrm{Oz}$ goes into Earth centre. In the given coordinates, the $3 \mathrm{D}$ plates spaces $\Omega$ read

$$
\begin{gathered}
\Omega_{1}=\left\{x, y, z /(x, y) \in[0, a] \times[0, b],-\frac{\delta_{1}}{2} \leq z \leq \frac{\delta_{1}}{2}\right\}, \\
\Omega_{2}=\left\{x, y, z /(x, y) \in[0, a] \times[0, b], \frac{1}{2}\left(\delta_{1}-\delta_{2}\right)+h_{1} \leq z \leq \frac{1}{2}\left(\delta_{1}+\delta_{2}\right)+h_{1}\right\},
\end{gathered}
$$

where the space $[0, a] \times[0, b]$ defines rectangular plate shape; $D_{1}, D_{2}$ are cylindrical plates 
stiffness. The governing equations are

$$
\begin{aligned}
& D_{1} \Delta^{2} w_{1}(x, y, t)+k \frac{E}{h} w_{1}(x, y, t) \Psi(x, y, t)=q_{1}^{*}+k \frac{E}{h}\left(w_{2}+h_{1}\right) \Psi(x, y, t), \\
& D_{2} \Delta^{2} w_{2}(x, y, t)+k \frac{E}{h} w_{2}(x, y, t) \Psi(x, y, t)=q_{2}^{*}+k \frac{E}{h}\left(w_{1}+h_{1}\right) \Psi(x, y, t),
\end{aligned}
$$

where the function

$$
\Psi=\frac{1}{2}\left[1+\operatorname{sign}\left(w_{1}-w_{2}-h_{1}\right)\right],
$$

and $\Psi=\Psi(x, y)$ plays a role of shells' contact space $\Omega^{*}$ indicator. Notice that if the initial plates location (clearance function) and the loads do not lead to a contact between plates during their deformations, then $\Psi \equiv 0$ and each of the plates vibrates independently. Otherwise, the governing equations are coupled.

The system (2.2) is associated with one of the following boundary conditions [6] on the boundary $\partial \Omega_{1}$,

$$
\begin{gathered}
\left.w_{i}\right|_{\partial \Omega_{i}}=\left.\frac{\partial w_{i}}{\partial n_{i}}\right|_{\partial \Omega_{i}}=0, \\
\left.w_{i}\right|_{\partial \Omega_{i}}=\left.\frac{\partial^{2} w_{i}}{\partial n_{i}}\right|_{\partial \Omega_{i}}=0, \quad(i=1,2) .
\end{gathered}
$$

Owing to D'Alembert principle, $q_{i}^{*}$ include both inertial and damping forces acting on the $i$ th plate of the form

$$
q_{i}^{*}(x, y, t)=q_{i}(x, y, t)-\frac{\gamma_{i}}{g} \frac{\partial^{2} w_{i}}{\partial t^{2}}-\varepsilon_{i} \frac{\partial w_{i}}{\partial t} .
$$

The system (2.2) is of high order with respect to time and spatial coordinates $(x, y)$. The computational process is as follows. In each time step, the following values are accounted from a previous step $\left.w_{i}\right|_{t_{k-1}}=w_{i}\left(x, y, t_{k-1}\right) ;\left.\dot{w}_{i}\right|_{t_{k-1}}=\dot{w}_{i}\left(x, y, t_{k-1}\right)$, and in order to improve a contact zone, the following iteration procedure for time $t_{k}$ is applied:

$$
\begin{aligned}
& D_{1} \Delta^{2} w_{1}^{(m+1)}+\frac{k E}{h_{1}} \Psi w_{1}^{(m+1)}=q_{1}^{* n}+\frac{k E}{h_{1}} \Psi\left(w_{2}^{(m)}+h_{1}\right), \\
& D_{2} \Delta^{2} w_{2}^{(m+1)}+\frac{k E}{h_{1}} \Psi w_{2}^{(m+1)}=q_{2}^{* n}+\frac{k E}{h_{1}} \Psi\left(w_{1}^{(m)}+h_{1}\right) .
\end{aligned}
$$

First PDEs (2.2) are reduced to the Cauchy problem through the second-order method of finite difference, then the problem is solved using fourth-order Runge-Kutta method. In each time step, the Gauss iteration procedure (2.7) is carried out, and the system order is reduced twice, which is important owing to computation time (see also [3]). Finishing the iteration procedure (2.7), the obtained values of $w_{i}$ and $\dot{w}_{i}$ serve as an initial condition for a next step of the Runge-Kutta method. The similar like methods have been also applied in $[1,2,4]$. The mentioned procedure allows for a contact zone improvement. Owing to the Runge principle, it has been found that the optimal step with respect to spatial coordinates is defined through a partition of space $\Omega_{j}$ into $15 \times 15$ parts, whereas 
time step is equal to $\Delta t=1 \cdot 10^{-3}$. In what follows, vibrations of two-layer plates with various boundary conditions along their contours are studied. The following four variants of the boundary conditions are accounted:

(i) two plates are clamped along their contours (boundary conditions (2.4));

(ii) two plates are ball-type supported (boundary conditions (2.5));

(iii) upper plate is clamped along its contour (boundary conditions (2.4)), whereas lower plate is ball-type supported along its contour (boundary conditions (2.5));

(iv) upper plate is ball-type supported along its contour (boundary conditions (2.5)), whereas lower one is clamped (boundary conditions (2.4)).

\section{Two plates are clamped along their contours (boundary conditions (2.4))}

Assume that two plates have same thickness $\left(\delta_{1}=\delta_{2}=\delta\right)$, and the frequency of excitation $\omega_{0}=\omega_{P}=9.973$, where $\omega_{0}$ is the frequency of a linear vibration of one-layer plate, $\varepsilon_{1}=\varepsilon_{2}=0.6$. The clearance between plates is $\overline{h_{1}}=h_{1} / \delta=0.007$ ( $\overline{h_{1}}$ is the nondimensional parameter). Both plates are subject to sinusoidal load of the form $q_{i}=Q_{i} \sin \omega p t$. Boundary conditions (2.4) are applied, and the initial conditions read

$$
\left.w_{1}\right|_{t=0}=\left.\dot{w}_{1}\right|_{t=0}=0
$$

Recall that in each time step, the iteration procedure (2.7) is applied. In Table 3.1, parts of time histories $w_{i}(0,5 ; 0,5 ; t), 26 \leq t \leq 32$, phase portraits $\dot{w}_{i}\left(w_{i}\right)$, and power spectrum $s(\omega)$ are presented only for lower plate, since the vibrations of lower plate are mirror reflections of the upper plate. Furthermore, the results are only given for the plate centre, since vibrations of other points are synchronized with those of the centre. During plates contact, there exist time instants where vibrations process is an unstationary one, then after a long time, it achieves a stationary state. For $q_{1}=-q_{2}<0.5$, the plates do not contact with each other, and harmonic vibrations occur.

This observation is confirmed through the following characteristics. In power spectrum, only one frequency is visible, and an ellipse occurs in the phase portrait. However, for $q_{1}=-q_{2}=2$ (a contact between plates occurred), a picture is changed: vibrations are no longer harmonic, and the phase portrait is of more complexity. Increasing the transversal load, a Hopf bifurcation occurs with a period tripling $\left(q_{1}=-q_{2}=11\right.$ ) (see $[8])$. Further, an interlace of vibrations on the excitation frequency and on the frequencies of period tripling and number eight is observed.

\section{Boundary conditions (2.5)}

Consider two-layer plate type construction with the same parameters as in the previously analyzed case, but with boundary conditions (2.5). Similarly as in the previous case, for $q_{1}=-q_{2}=0.5$, harmonic vibrations occur. However, now bifurcations appear already for $q_{1}=-q_{2}=1.5$. Increasing $q_{i}$, a picture of plates bending becomes more complicated. For $q_{1}=-q_{2}=3$, period tripling occurs, then a transition to chaos takes place $\left(q_{1}=-q_{2}=\right.$ 4). This is clearly expressed in both phase portrait and power spectrum. For $q_{1}=-q_{2}=5$, again Hopf bifurcation appears, then its collapse takes place $\left(q_{1}=-q_{2}=6\right)$. The scenario is composed of interlace of Hopf bifurcations and a transition into harmonic vibrations. 
428 Novel procedure to compute a contact zone magnitude

Table 3.1

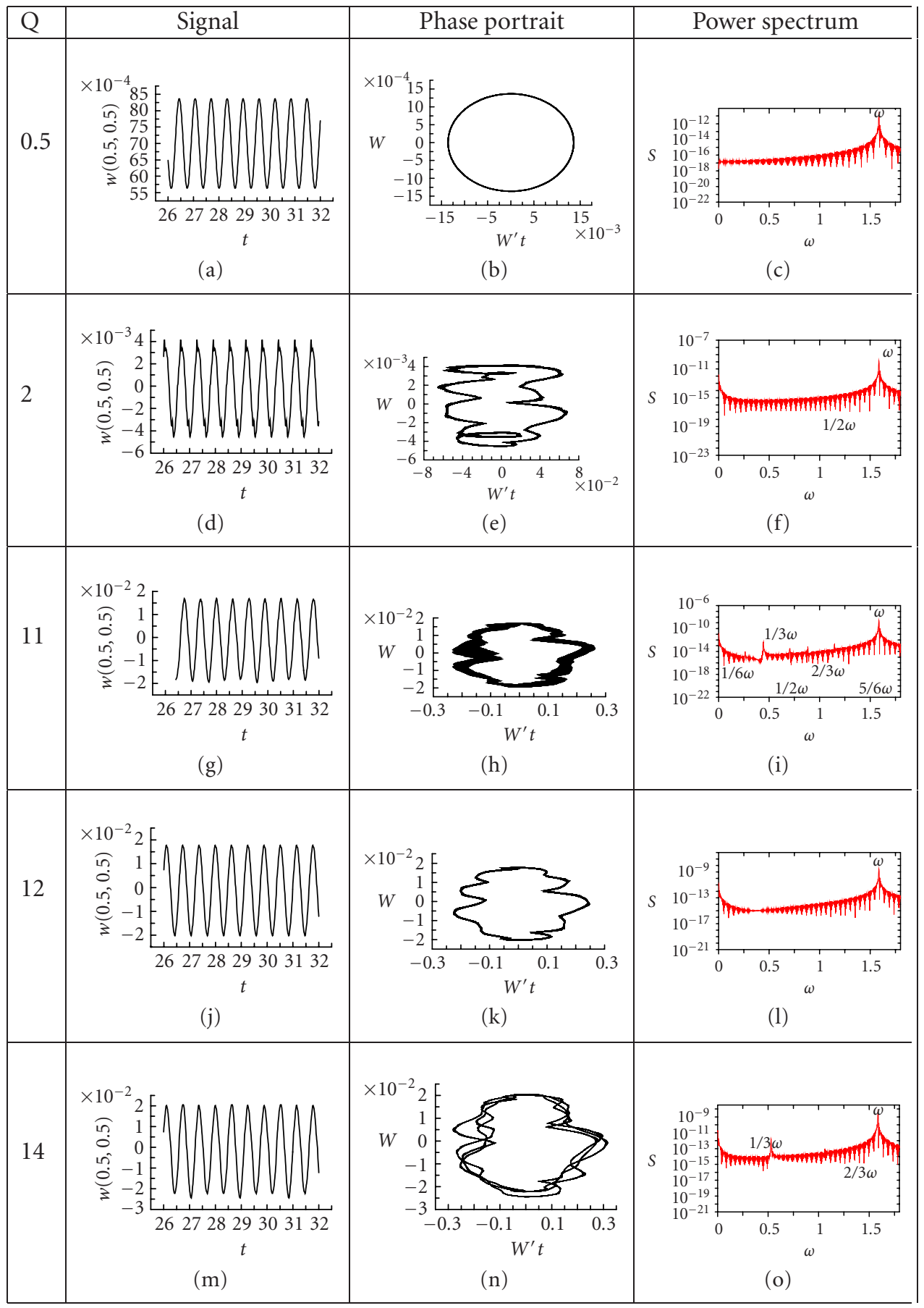


Table 3.1. Continued.

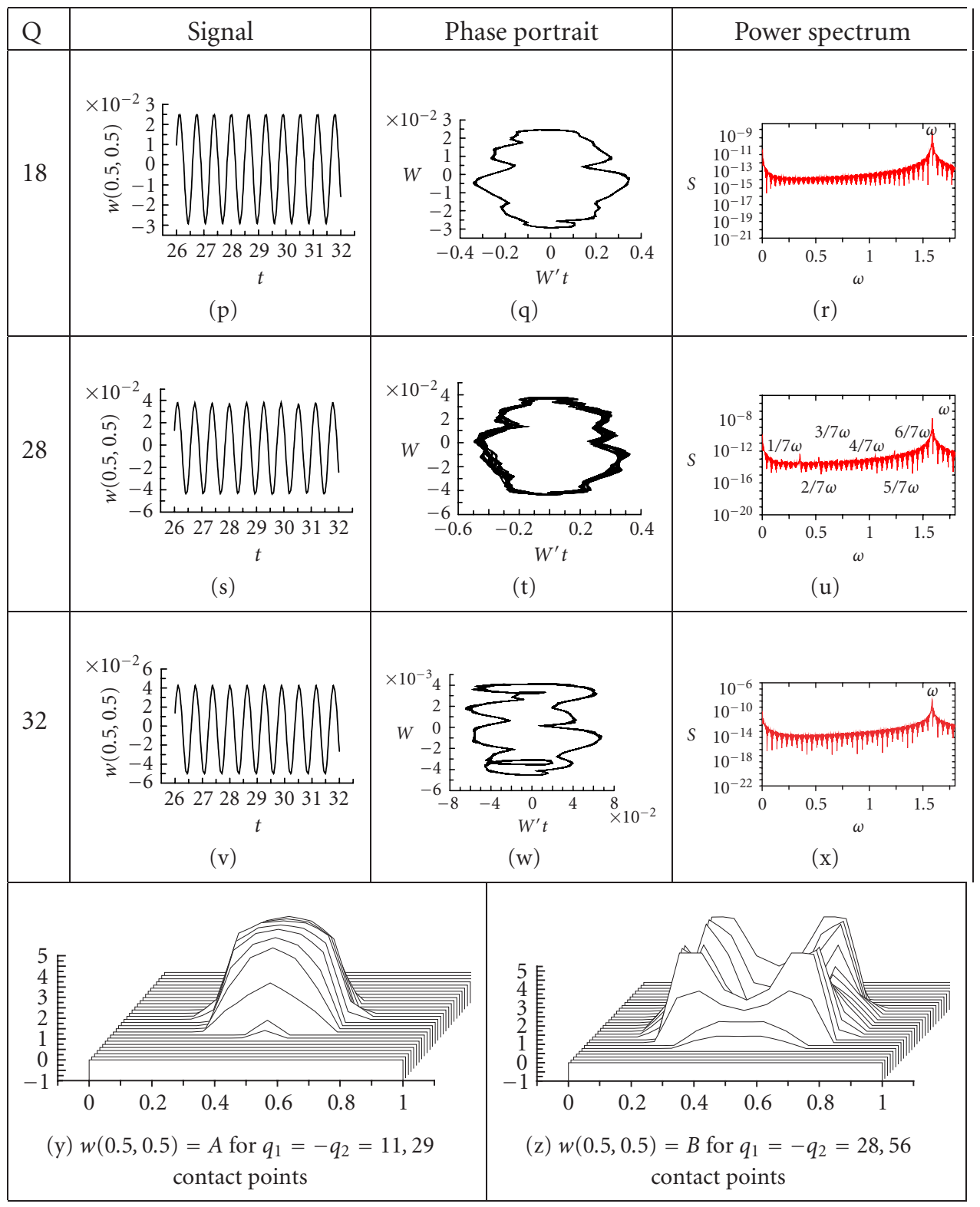

\section{Vibrations associated with different boundary conditions}

In Table 5.1, same characteristics as in Table 5.2 are reported, but now the upper layer is ball-type supported along the contour (boundary conditions (2.5)), whereas the lower layer is clamped (boundary conditions (2.4)).

For $q_{1}=-q_{2}=0.5$, vibrations of two plates are harmonic, and each of the plates vibrates with its own frequency. For $q_{1}=-q_{2}=1.5$, when a contact between plates occurs, 
430 Novel procedure to compute a contact zone magnitude

Table 5.1

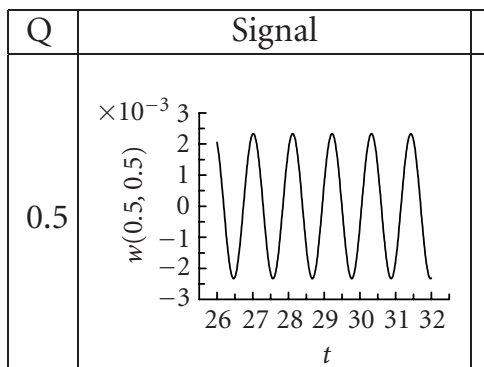

(a)

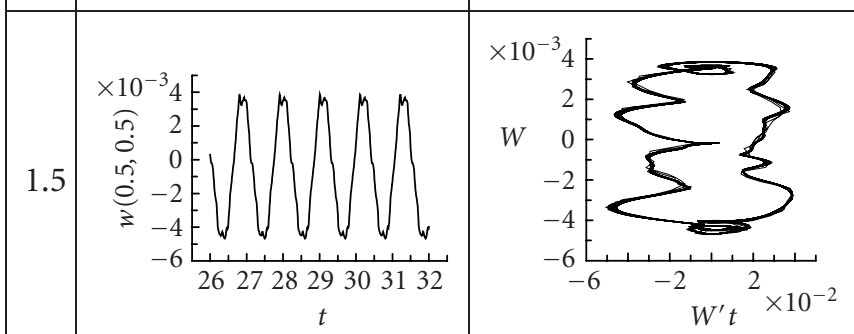

(d)

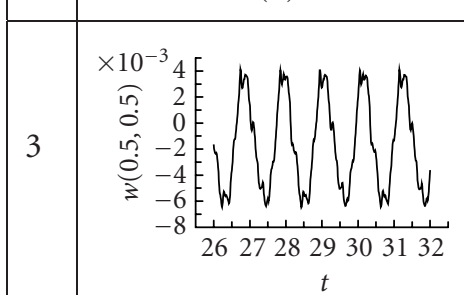

(g)

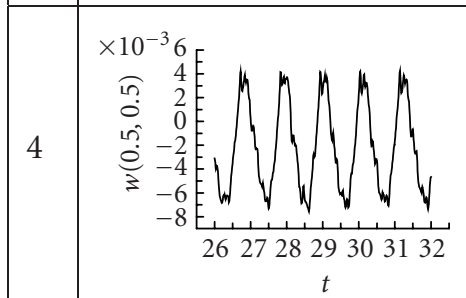

(j)

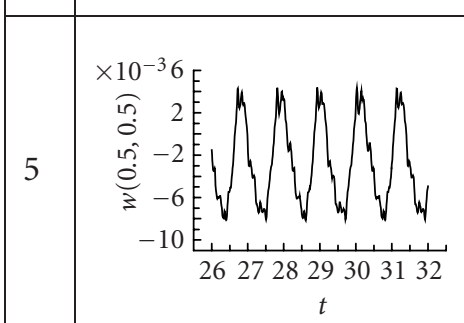

(m)

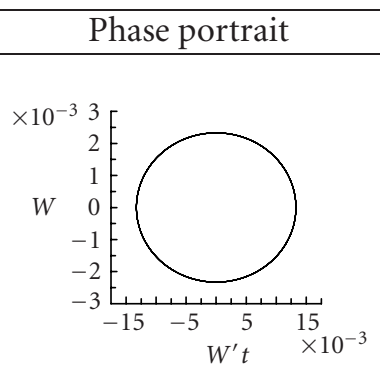

(b)

(e)

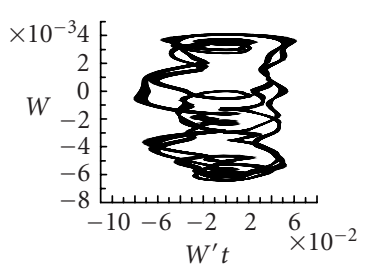

(h)

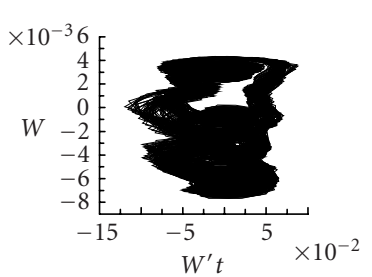

(k)

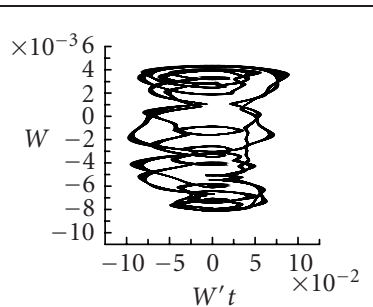

(n)
Power spectrum

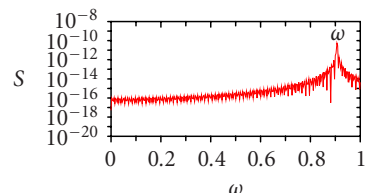

(c)

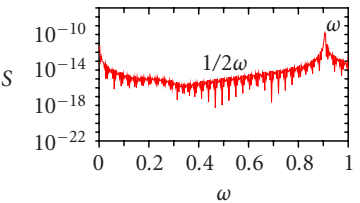

(f)

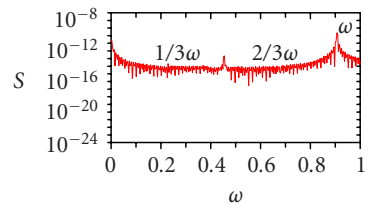

(i)

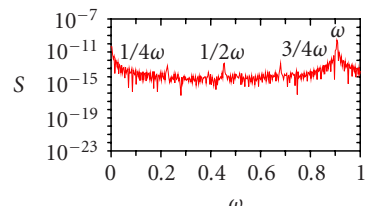

(1)

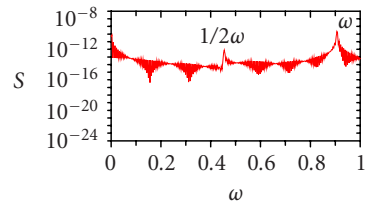

(o) 
Table 5.1. Continued.

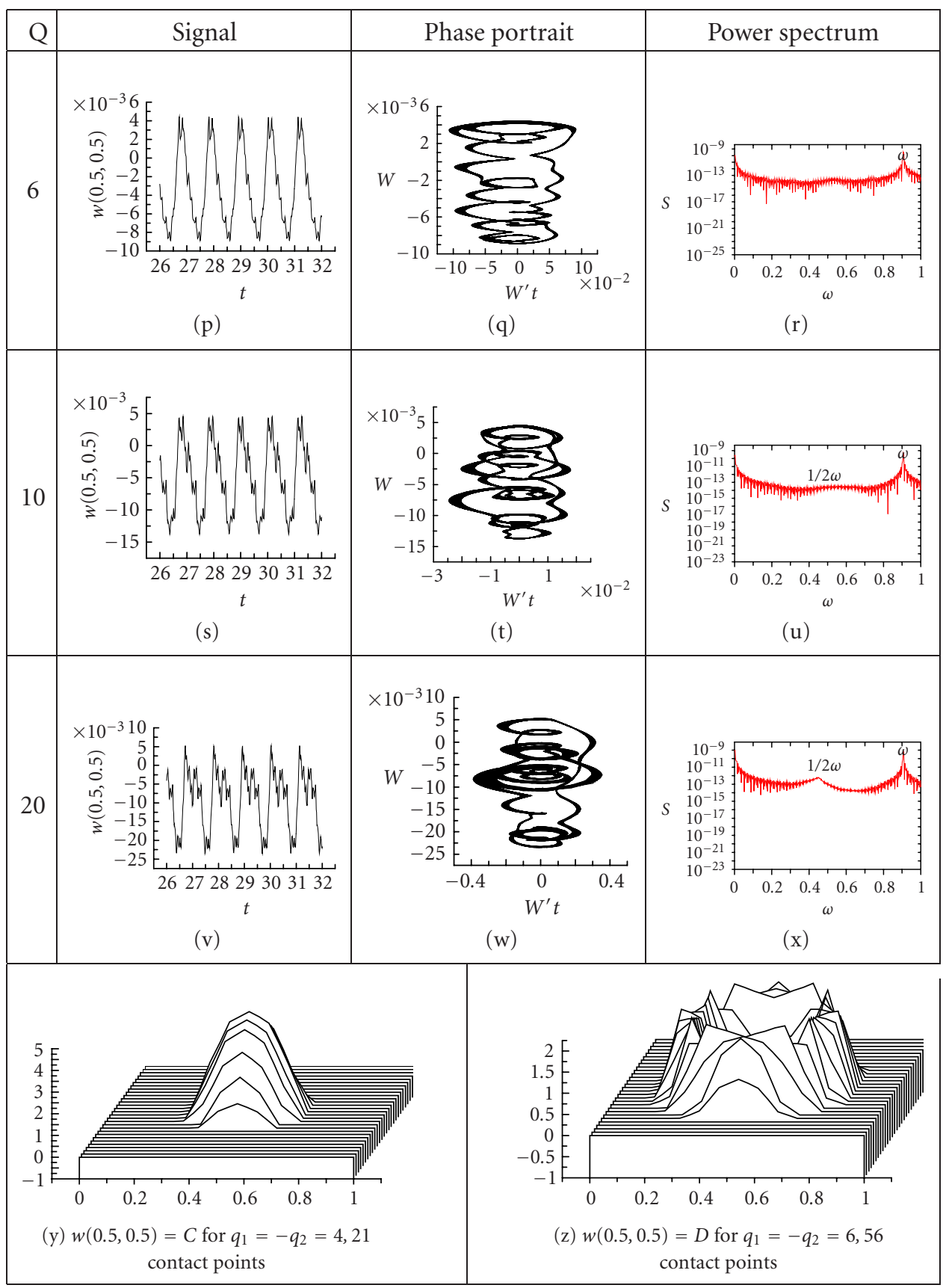


432 Novel procedure to compute a contact zone magnitude

Table 5.2

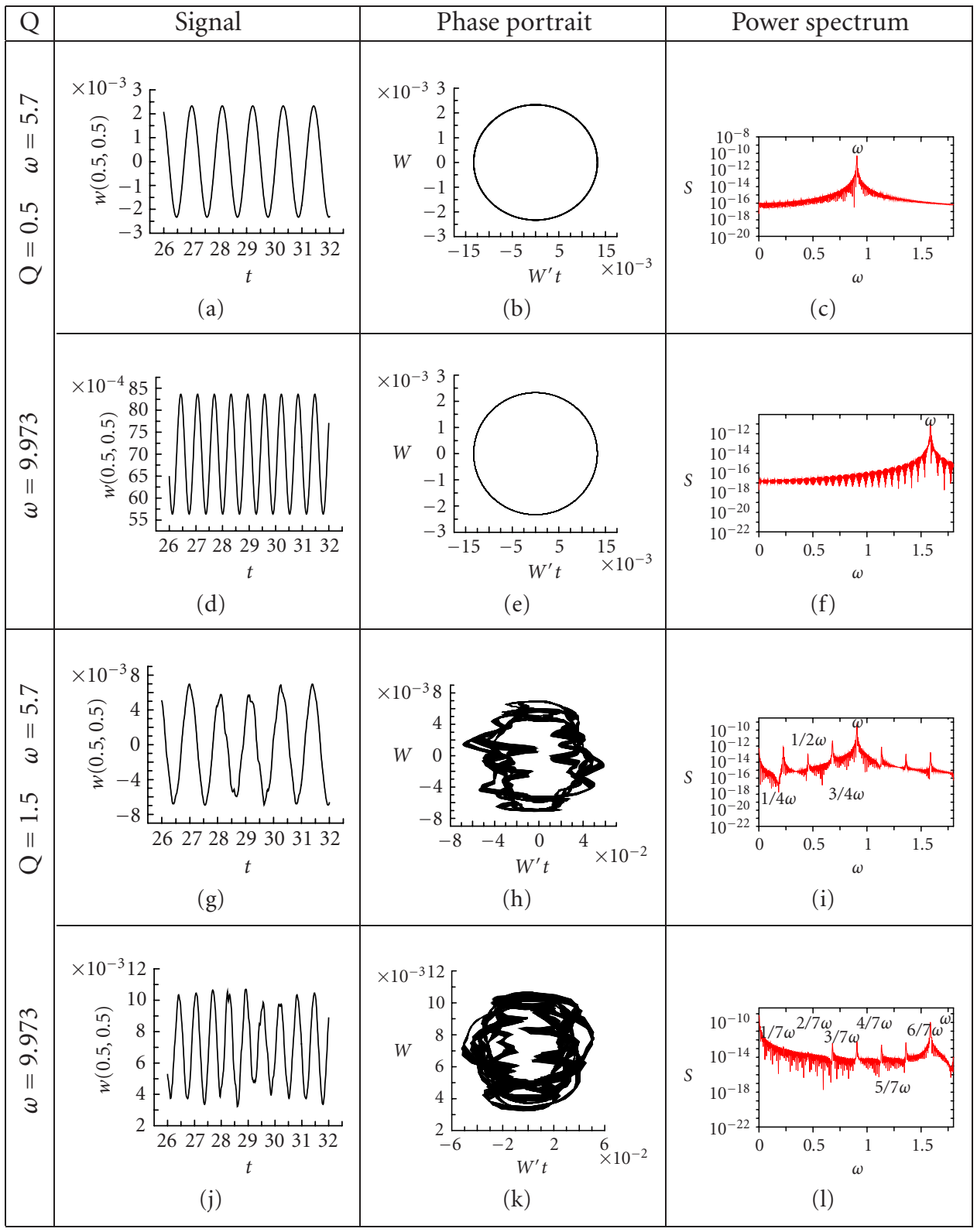

in upper-plate power spectrum, two Hopf bifurcations are remarkable, whereas period seven is associated with lower plate (see the power spectrum). For $q_{1}=-q_{2}=3$, in the power spectrum of lower plate, also two Hopf bifurcations appear, and two plates begin to vibrate with one frequency.

In Table 5.3, the same characteristics as in Table 5.1 are reported, but for different boundary conditions. Namely, upper plate is clamped through its contour (boundary 
Table 5.2. Continued.

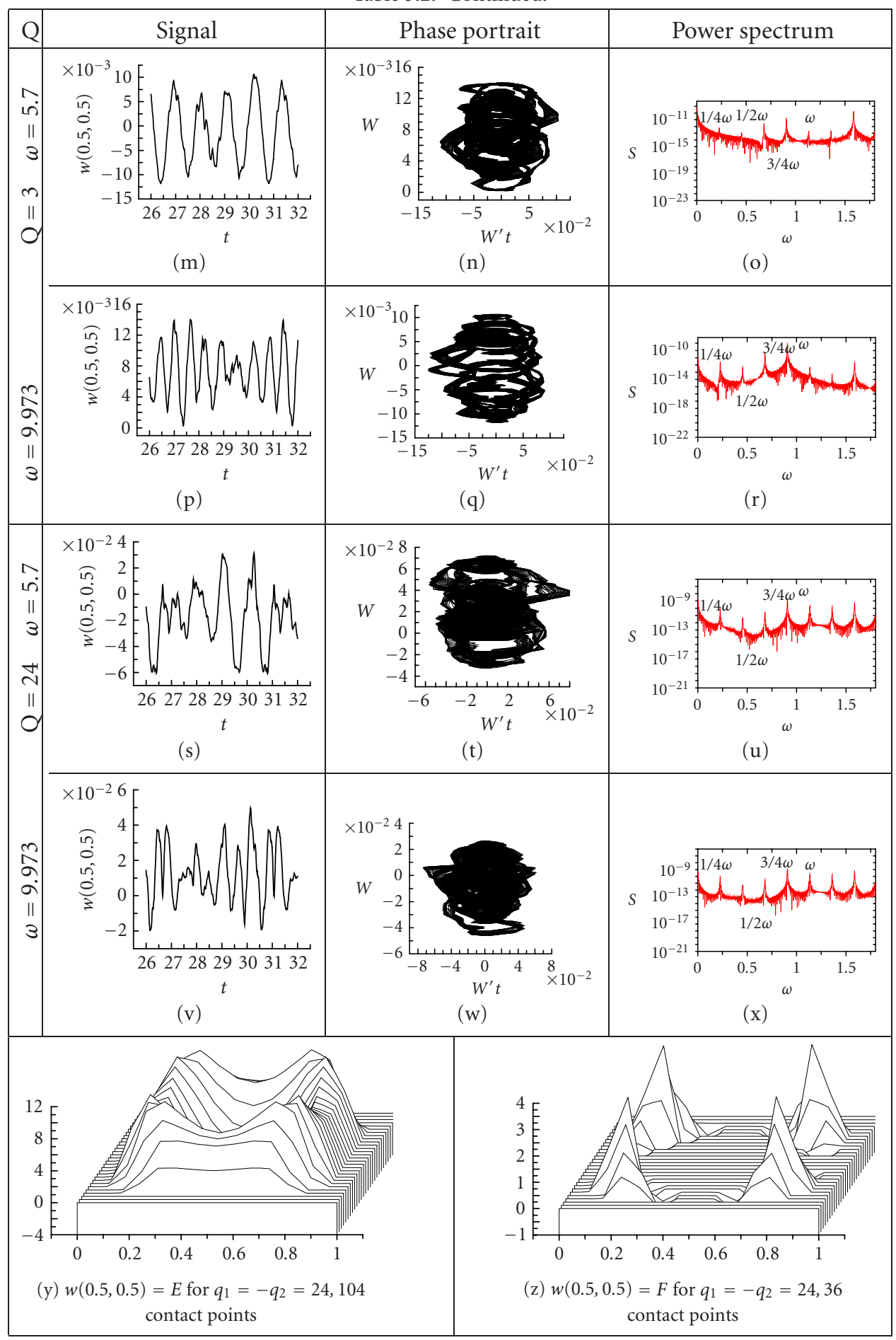


434 Novel procedure to compute a contact zone magnitude

Table 5.3

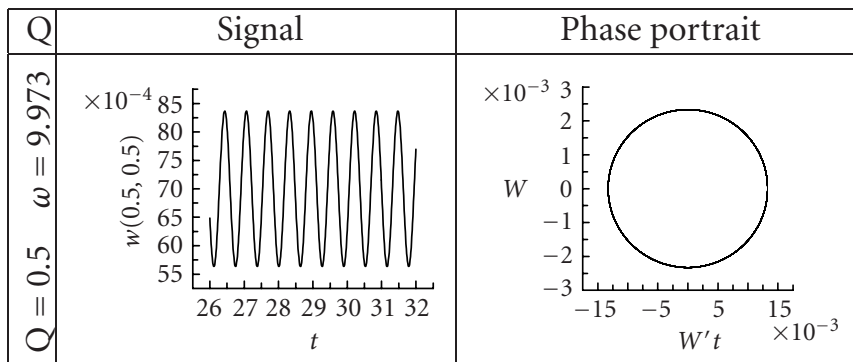

(a) (b)

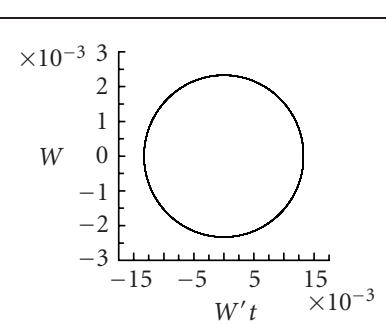

(e)
Power spectrum

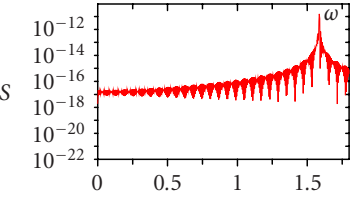

(c)

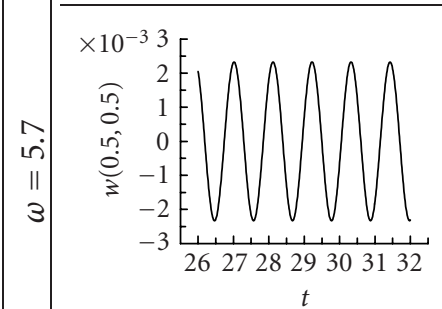

(d)

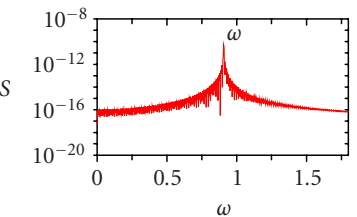

(f)

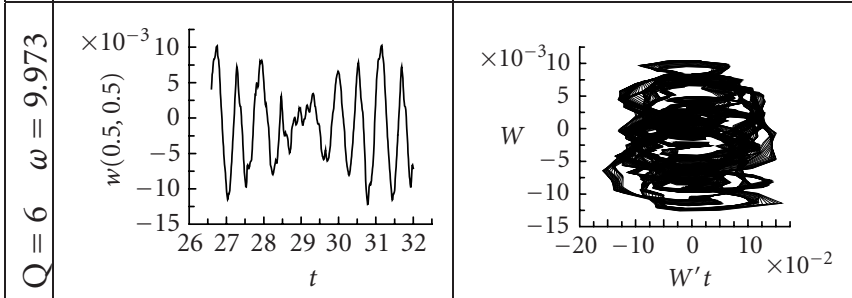

(g) (h)

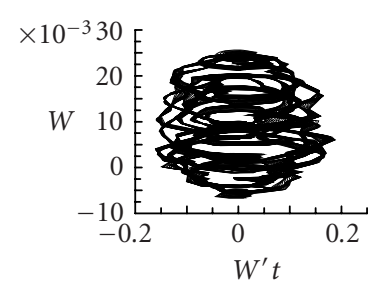

$(\mathrm{k})$

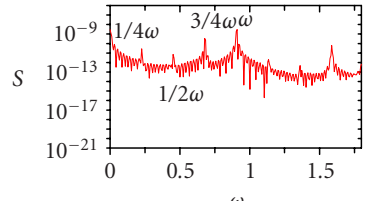

(1)

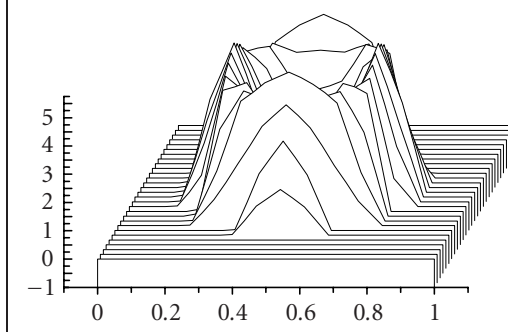

(m) $w(0.5,0.5)=K$ for $q_{1}=-q_{2}=6,61$ contact points

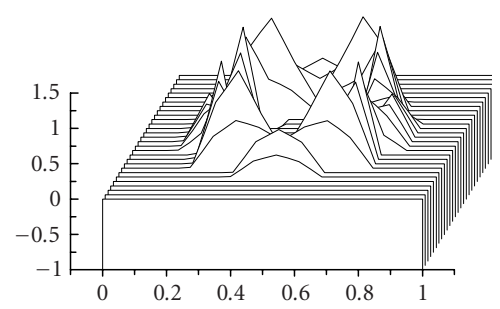

(n) $w(0.5,0.5)=M$ for $q_{1}=-q_{2}=6,40$ contact points 
conditions (2.4)), whereas the lower plate is supported through the boundary conditions (2.5).

For $q_{1}=-q_{2}=0.5$, both plates vibrations are harmonic. For $q_{1}=-q_{2}=6$, vibrations of both plates are synchronized with one frequency. Then two Hopf bifurcations follow.

\section{Conclusions}

The carried out analysis exhibits complex vibrations of two-layer system of plates: series of Hopf bifurcations occurs, where periods three, five, and seven Hopf bifurcations are exhibited. The detected bifurcations in our complex system have been theoretically predicted by Sharkovskiy while analyzing the logistic curves [8]. It should be emphasized that contact load value depends essentially on the number of contacting points.

Some novel dynamical phenomena have been detected. For example, if the upper plate is ball-type supported, and the lower one is clamped along its contour, synchronization takes place. Namely, both plates start to vibrate with the same fundamental frequency $\omega=1.6$ (a ball frequency) earlier than in the case of clamping and ball-type supports. After the occurring synchronization, further increase of loading has not changed dynamics qualitatively.

\section{References}

[1] J. Awrejcewicz and A. V. Krysko, Analysis of complex parametric vibrations of plates and shells using Bubnov-Galerkin approach, Archive of Appl. Mech. 73 (2003), 495-504.

[2] J. Awrejcewicz and V. A. Krysko, Nonclassical Thermoelastic Problems in Nonlinear Dynamics of Shells, Scientific Computation, Springer-Verlag, Berlin, 2003.

[3] J. Awrejcewicz, V. A. Krysko, and A. V. Krysko, On the economical solution method for a system of linear algebraic equations, Math. Probl. Eng. 2004 (2004), no. 4, 377-410.

[4] J. Awrejcewicz, V. A. Krysko, and G. G. Narkaitis, Bifurcations of a thin plate-strip excited transversally and axially, Nonlinear Dynam. 32 (2003), no. 2, 187-209.

[5] B. Ya. Kantor, Contact Problems of Nonlinear Shells Theory, Naukova Dumka, Kiev, 1990.

[6] M. S. Kornishin, Nonlinear Problems of Plates and Shallow Shells and Methods of Their Solutions, Nauka, Moscow, 1964.

[7] A. V. Krysko, V. A. Krysko, V. A. Ovsiannikova, and T. B. Babenkova, Complex vibrations of twolayer uncoupled plates subject to longitudinal sinusoidal load, Izv. Vuz., Striotel'stvo (2002), no. 6, 23-30 (Russian).

[8] A. N. Sharkovskiy, Existence of cycles exhibited by continuous mapping of a straight line into itself, Ukrainian Math. J. 16 (1964), no. 1, 61-71 (Russian).

J. Awrejcewicz: Department of Automatics and Biomechanics, Faculty of Mechanical Engineering, Technical University of Lodz, 1/15 Stefanowskiego Street, 90-924 Lodz, Poland

E-mail address: awrejcew@p.lodz.pl

V. A. Krysko: Department of Mathematics, Saratov State University, B. Sadovaya 96a, 410054 Saratov, Russia

E-mail address: tak@san.ru

O. Ovsiannikova: Department of Mathematics, Saratov State University, B. Sadovaya 96a, 410054 Saratov, Russia

E-mail address: tak@san.ru 


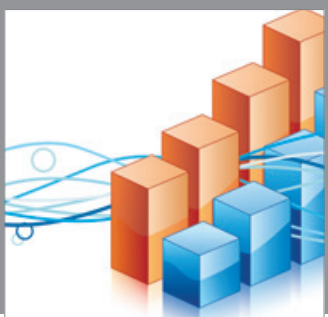

Advances in

Operations Research

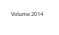

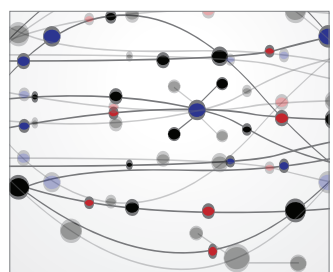

\section{The Scientific} World Journal
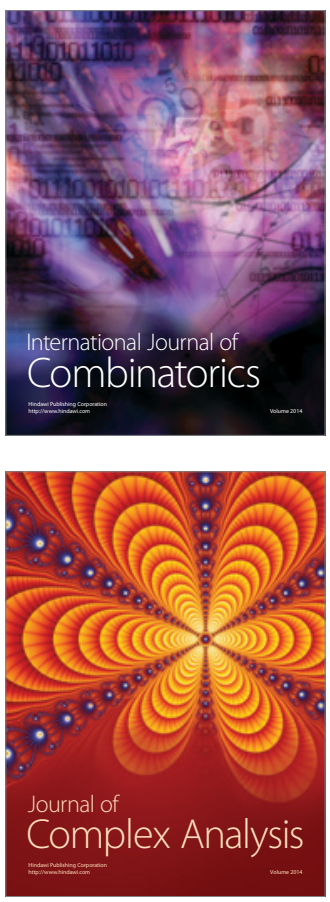

International Journal of

Mathematics and

Mathematical

Sciences
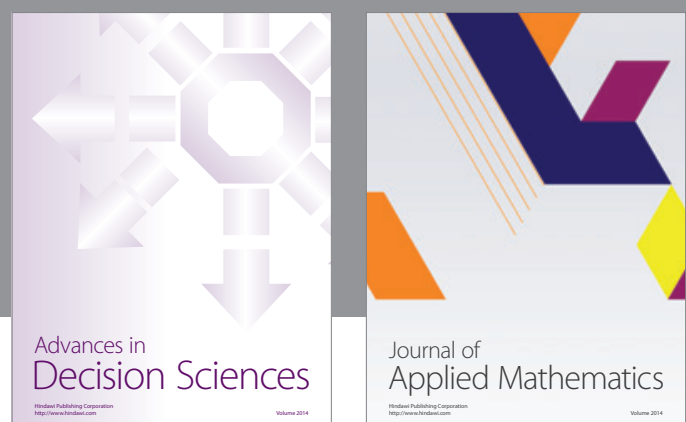

Journal of

Applied Mathematics
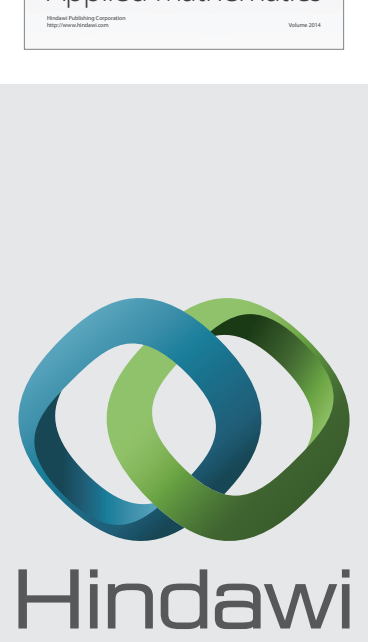

Submit your manuscripts at http://www.hindawi.com
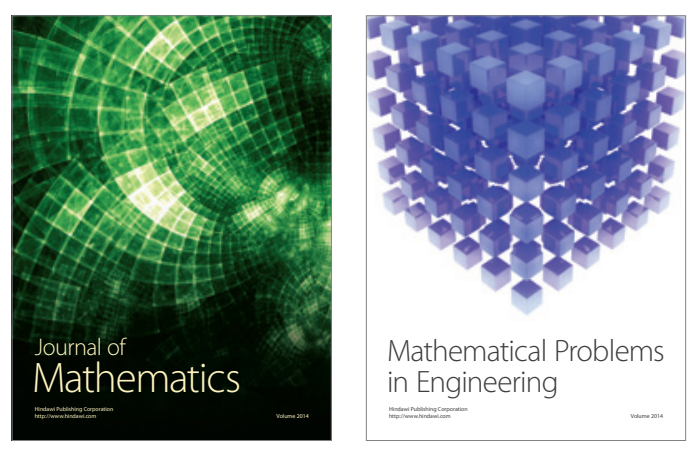

Mathematical Problems in Engineering
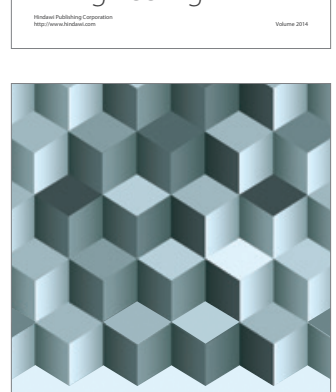

Journal of

Function Spaces
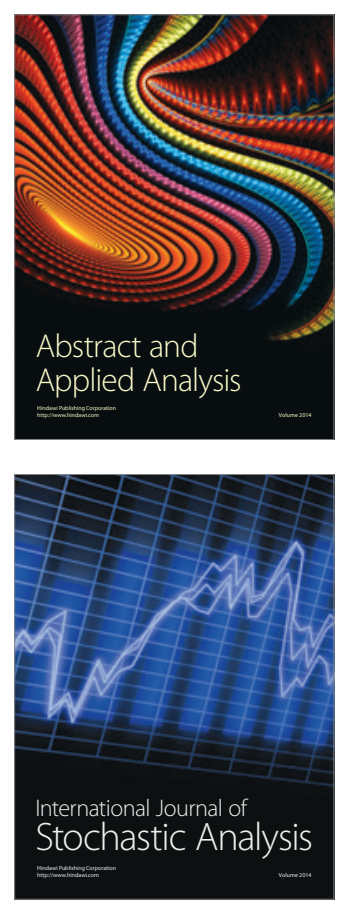

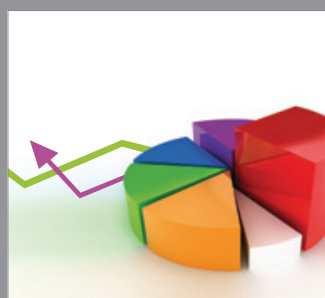

ournal of

Probability and Statistics

Promensencen
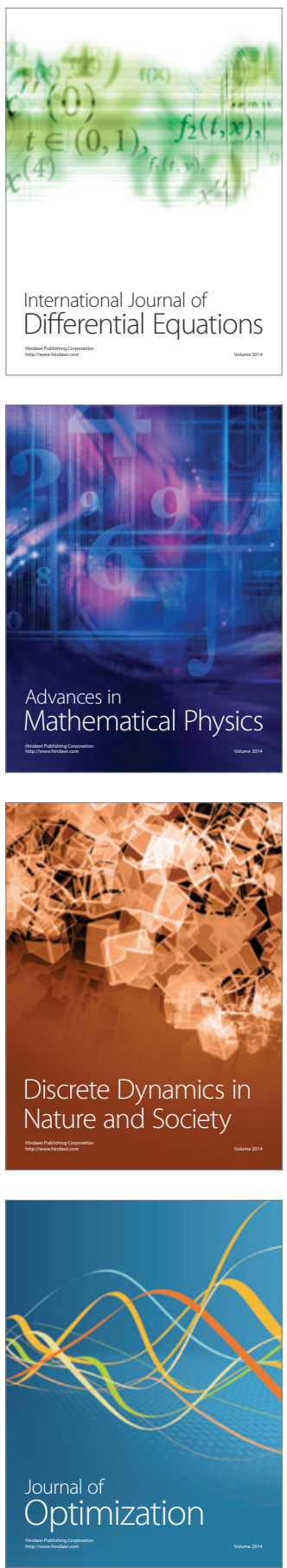